\title{
PROPRIEDADES ESPECTRAIS DA MATÉRIA ORGÂNICA LEVE-LIVRE E LEVE INTRA-AGREGADO DE DOIS LATOSSOLOS SOB PLANTIO DIRETO E PREPARO CONVENCIONAL ${ }^{(1)}$
}

\author{
A. A. FREIXO(2), L. P. CANELLAS ${ }^{(3)}$ \& P. L. O. A. MACHADO $(4)$
}

\begin{abstract}
RESUMO
Este trabalho teve como objetivo estudar a natureza química das frações leves-livres (FLL) e leves intra-agregado (FLI) da matéria orgânica do solo, obtidas pelo fracionamento físico do solo por densidade, por meio da espectroscopia na região do infravermelho, para verificar se tais frações constituem compartimentos distintos da matéria orgânica do solo. Foram anali sadas amostras de Latossolos de dois estados do Brasil (RS e GO), submetidos a plantio direto e preparo convencional, em distintos sistemas de rotação de culturas. A análise por infravermel ho revelou diferenças contrastantes entre os compartimentos orgânicos estudados. Os espectros de IV da fração leve-livre apresentaram confi guração semel hante aos dos resíduos vegetais, indicando que ela se encontra em estádios iniciais de transformação. Não foram observadas diferenças estruturais na $F L L$ entre os distintos sistemas de preparo e rotação de culturas. Os espectros de IV da FLI apresentaram bandas de absorção N-H e C-O de polissacarídeos menos intensas e em maior conjugação, em relação aos espectros da FLL, características de material mais humificado. Foi observada ainda uma maior transformação estrutural da fração leve intra-agregado em solos sob preparo convencional, quando comparada à $F L I$ de solo sob vegetação natural e plantio direto. Os índices de hidrofobicidade (IH) e de condensação (IC), determinados a partir de relações entre as bandas de absorção de grupamentos $-\mathrm{CH}_{3}$ alifáticos, $\mathrm{C}-\mathrm{O}$ de polissacarídeos $\mathrm{C}=0$ conjugados, permitiram identificar as diferenças na recalcitrância e condensação das frações leves. Constatou-se que ambos os índices foram significativamente maiores para a matéria orgânica intra-agregado, por conseqüência de seu maior grau de humificação.
\end{abstract}

Termos de indexação: fracionamento do solo por densidade, solos cultivados e sob floresta, espectroscopia de infravermelho.

\footnotetext{
(1) Parte da Tese de Mestrado do primeiro autor, apresentada ao Curso de Pós-Graduação em Agronomia - Ciência do Solo, Departamento de Solos, U niversidade Federal Rural do Rio de J aneiro - UFRRJ . Recebido para publicação em outubro de 2000 e aprovado em maio de 2001.

(2) Bióloga, MScAgronomia - Ciência do Solo, Universidade Federal Rural do Rio deJ aneiro- UFRRJ . BR 467, Km 7, CEP 23890-000 Seropédica (RJ).

(3) Professor Visitante da Universidade Estadual do Norte Fluminense - UENF. Av. Alberto Lamego 2000, CEP 28015-620 Campos dos Goytacazes (RJ)

(4) Pesquisador da Embrapa Solos. Rua J ardim Botânico 1024, CEP 22460-000 Rio de J aneiro (RJ ).
} 


\author{
SUMMARY: SPECTRAL PROPERTIES OF FREE LIGHT AND INTRA- \\ AGGREGATE ORGANIC MATTER OF TWO OXISOLS UNDER \\ NO-TILLAGE AND CONVENTIONAL TILLAGE
}

\begin{abstract}
The objective of this study was to investigate the chemical nature of free (FLF) and intra-aggregate (ILF) light fraction of soil organic matter, using infrared spectroscopy as well as to determine whether these fractions are compositionally distinct pools of soil organic matter. Both FLF and ILF were isolated by flotation on $\mathrm{Nal}$ solution at a specific gravity of 1.8; for ILF extraction, thesoil samplewas sonicated after FLF isolation. Samples were collected from two Oxisols from contrasting regions of Brazil (southern and central). Treatments consisted of no-tillage and conventional tillage (ploughing followed by light discharrowings), under different crop rotation systems. FLF spectra showed featuressimilar to that of plant debris, indicating that these fractions are at early transformation stages. Nostructural differencewas found in theFLF among thedifferent tillageand crop rotation systems. Compared to the FLF spectra, the ILF spectra showed less intense N-H and C-O adsorption bands and werein greater conjugation, which is characteristic of a morehumified material. The ILF of soils under conventional tillage presented morechanges than the ILF of soils under native vegetati on and no tillage. The hydrophobicity $(H R)$ and condensation (CR) indexes, determined by there ationship between theabsorption bands of al ifatic $-\mathrm{CH}_{3}$, polysacharide $\mathrm{C}-\mathrm{O}$ and conjugated $\mathrm{C}=\mathrm{O}$ functional groups, led to the identification of differences in recalcitrance and condensation of the light fractions. Both hydrophobicity and condensation indexes weresignificantly higher for ILF than for FLF, dueto theformer's greater moisture degree
\end{abstract}

Index terms: organic matter, density fractionation, cultivated and forest soils, infrared spectroscopy.

\section{INTRODUÇÃO}

O estudo da transformação da matéria orgânica do solo (MOS) por meio do uso do fracionamento físico vem crescendo nos últimos anos, embora haja registros no final do século passado do uso do fracionamento granulométrico do solo para investigar a MOS (Christensen, 1992; Feller, 1999). As frações da MOS, presentes em diferentes compartimentos, podem apresentar diferentes velocidades de decomposição, dependendo da sua localização física no solo. Sohi et al. (2001) desenvolveram um método de fracionamento da MOS que extrai, seqüencialmente, numa mesma amostra, a matéria orgânica entre agregados e dentro deles (frações leves) e aquela associada às partículas de argila, silte e areia (frações pesadas).

No Brasil, alguns estudos envolvendo o fracionamento físico da MOS já foram apresentados, contudo o procedimento adotado compreendeu apenas a quantificação da matéria orgânica associada às partículas minerais do solo (Silva et al ., 1999; Freitas et al ., 2000). Skjemstad et al . (1986) constataram diferenças estruturais entre a fração leve e a fração orgânica associada à areia, demonstrando a importância da distinção entre as frações leves e pesadas pel ofracionamentofísico. Tais resultados são importantes, principal mentequando se busca quantificar os diferentes compartimentos orgânicos, com vel ocidades de transformação e características funcionais distintas, utilizados no desenvolvimento de modelos simuladores da dinâmica da MOS (Parton et al., 1987).

A maior parte da MOS ocorre associada às partículas minerais do solo, considerada um compartimentoaltamenteresistenteà transformação ou ao ataque mi crobiano (Stevenson, 1994; Feller \& Beare, 1997). Pequena proporção da MOS, constituída principalmente por resíduos vegetais, pode estar livreou presente no interior deagregados instáveis, denomi nada fraçãol eve-livre, e dentro de agregados estáveis, denominada fração leve intra-agregado.

SegundoTheng et al . (1989) eChristensen (1996), a fração leve-livre, também denominada de matéria macrorgânica, matéria orgânica particulada ou matéria orgânica não complexada, pode estar livre ou não firmemente associada com os minerais do solo. A fração leve intra-agregado, também denominada de matéria orgânica particulada intraagregado (Six et al., 2000) ou matéria orgânica não complexada oclusa (Christensen, 1996), está oclusa em agregados do solo, mas não está fortemente associada aos minerais do solo. Freixo (2000) observou que aproximadamente $60 \%$ do carbono orgânico total de um horizonte superficial $(0-5 \mathrm{~cm})$ de um Latossolo Vermel ho-E scuro de Passo F undo (RS), sob floresta, encontravam-se na fração pesada, $37 \%$ na fração leve-livre e 3 \% na fração intra-agregado. 
Onze anos de cultivo do solo resultaram em um aumento na proporção da MOS associada às partículas deargila, siltee areia (aproximadamente 90 \% do carbono orgânico total).

Embora a composição da MOS seja bastante complexa e variável, o estudo espectroscópico das frações leves pode ser útil na identificação de diferenças entre os dois compartimentos da MOS leve, decorrentes dos processos de decomposição e proteção física dos resíduos orgâni cos (Golchin et al., 1994b). Pode-se avaliar também a influência do manejo do sol o sobre a natureza química das frações leves (Skjemstad et al., 1986; Wander \& Traina, 1996).

A espectroscopia de infravermel ho (IV) baseiase na propriedade vibratória inerente aos átomos de uma molécula. Cada molécula responde de forma diferente à radiação, o que proporciona diferentes bandas de absorção no espectro infravermel ho (Martin-N eto et al., 1996). Os espectros de infravermelho fornecem informações sobre a estrutura dos grupos funcionais presentes na matéria orgânica, bem como sobre a natureza desuas ligações químicas esua reatividade (Stevenson, 1994; J ohnston et al., 1994; J ohnston \& Aochi, 1996).

Embora a espectroscopia de IV tenha sido inicial mente utilizada de modo qual itativo, pel ofato de a técnica seguir os princípios da Lei de BeerLambert, aplicações quantitativas foram apresentadas por Schnitzer (1971).

Recentemente, com a disponibilidade da espectroscopia de IV e a transformada de Fourier (IV-TF), foi possível obter espectros de substâncias húmi cas muito mais preci sos e com mel hor definição. Assim, aumentou-se a possibilidade de aplicação quantitativa da espectroscopia deIV, correlacionandose estreitamentecom outros métodos espectroscópicos (ex. ressonância magnética nuclear) e potenciomé tricos (Cabassis, 1991; Celi et al., 1997). É possível obter determinações quantitativas através do IV-TF com al gumas vantagens em rel ação a outros métodos em virtude do menor tempo de aquisição dos espectros e das quantidades menores de amostras (Davis et al., 1999).

É importante ressaltar que as determinações quantitativas devem ser relativizadas com a introdução de um padrão interno (ex. mistura de uma quantidade exata em mol $\mathrm{CH}_{3} \mathrm{~L}^{-1}$ para leitura em $2.930 \mathrm{~cm}^{-1}$ ou de $\mathrm{COOH}$ para leituras em $1.720 \mathrm{~cm}^{-1}$ ) ou entre amostras quando se compara a relação de absorbâncias (I nbar et al. 1989).

Este trabalho teve como objetivo estudar, através da espectroscopia de IV-TF, a natureza química das frações leves-livres e intra-agregado, obtidas pelo fracionamento físico por densidade, de modo a verificar se tais frações constituem compartimentos distintos da MOS e identificar possíveis alterações em sua estrutura química, decorrentes dos diferentes tipos de preparo do solo e rotações de culturas, em Latossolos do Cerrado e do Sul do Brasil.

\section{MATERIAL E MÉTODOS}

Para este estudo, amostras de solo foram coletadas de um Latossolo Vermelho distroférrico (antigo Latossolo Vermelho-Escuro distrófico), localizado no Campo Experimental da Embrapa Trigo, no município de Passo Fundo (RS), e de um Latossolo Vermel ho-Amarelo, localizado no Campo Experimental da E mbrapa Arroz e Feijão, em Santo Antônio de Goiás (GO). A área da Embrapa Trigo apresenta precipitação anual média de $1.746 \mathrm{~mm}$ e temperatura anual média de $19,4^{\circ} \mathrm{C}$. A área da E mbrapa Arroz eFeijão apresenta uma estação seca pronunciada de março a setembro. A precipitação anual média é de 1.500 mm e a temperatura média anual é de $23^{\circ} \mathrm{C}$.

O experimento em blocos $(n=3)$ casualizados com parcelas subdividadas na Embrapa Trigo foi instalado em 1987. Cada parcela (tipo de preparo do solo) continha subparcelas (sistemas de cultivo) dispostas ao acaso. Os dois tipos de preparo do solo foram: (a) plantio direto (PD) e (b) preparo convencional (aração, utilizando arado de discos, seguida de gradagens leves - PC). Cada tipo de preparo continha dois sistemas de cultivo: (a) sucessão trigo/soja (T/S) e (b) rotação trigo/soja e ervilhaca/milho (T/S-E/M). Cada parcela media $360 \mathrm{~m}^{2}(4 \times 90 \mathrm{~m})$.

Na Embrapa Arroz e Feijão, o experimento de 1995 apresentava duas faixas (plantio direto e plantio convencional), e, em cada faixa, os sistemas de cultivo foram dispostos ao acaso em três repetições. Os tipos de preparo do solo consistiam de: (a) plantio direto (PD) e (b) plantio convencional (aração com arado de aivecas, seguida de duas gradagens leves niveladoras - PC). Cada tipo de preparo do solo continha os seguintes sistemas de cultivo: (a) pousio/arroz e pousio/soja (P/A-P/S) e (b) crotalária/arroz e milheto/soja (Cr/A-Mi/S). Cada parcela media $132 \mathrm{~m}^{2}$ (3,3 x 40,0 m).

Nas áreas estudadas, havia ainda remanescentes da vegetação natural (floresta secundária), adjacentes aos sítios experimentais. São elas: em Passo F undo, a Floresta Tropical Subperenifólia, e em Santo Antônio de Goiás, a vegetação de Cerrado. As princi pais características quími cas efísicas dos dois sol os estudados são apresentadas no quadro 1.

Os solos foram amostrados no Campo Experimental da Embrapa Trigo em dezembro de 1998, logo após a colheita do trigo. No Campo Experimental da Embrapa Arroz e Feijão, a amostragem foi real izada em junho de 1999, durante o pousio, na rotação $\mathrm{P} / \mathrm{S}-\mathrm{P} / \mathrm{A}$, e durante o cultivo de crotalária, na rotação $\mathrm{Cr} / \mathrm{A}-\mathrm{Mi} / \mathrm{S}$. As amostras de 
Quadro 1. Caracterização química e física de amostras superficiais de Latossolo Vermelho distroférrico, Passo F undo (RS) e Latossolo Vermelho-Amarelo, Santo Antônio de Goiás (GO)

\begin{tabular}{|c|c|c|c|c|c|c|c|c|c|c|c|c|c|}
\hline \multirow{3}{*}{$\begin{array}{l}\text { Sistema de } \\
\text { cultivo(1) }\end{array}$} & \multicolumn{9}{|c|}{ Propriedade química } & \multicolumn{4}{|c|}{ Granulometria } \\
\hline & \multirow{2}{*}{ pH $\mathbf{H}_{2} \mathrm{O}$} & \multirow{2}{*}{$\mathbf{A l}^{3+}$} & \multirow{2}{*}{$\mathbf{H}+\mathbf{A l}$} & \multirow{2}{*}{$\mathrm{Ca}^{2+}$} & \multirow{2}{*}{$\mathbf{M g}^{2+}$} & \multirow{2}{*}{$\mathbf{K}^{+}$} & \multirow{2}{*}{ СТC } & \multirow{2}{*}{$\mathbf{v}$} & \multirow{2}{*}{$\mathbf{P}$} & \multicolumn{2}{|c|}{ Areia } & \multirow{2}{*}{ Silte } & \multirow{2}{*}{ Argila } \\
\hline & & & & & & & & & & Grossa & Fina & & \\
\hline & & & 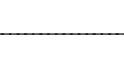 & \multicolumn{2}{|c|}{$-\mathrm{cmol}_{\mathrm{c}} \mathrm{dm}^{-3}$} & & - & $\%$ & $\mathrm{~g} \mathrm{~kg}^{-1}$ & & - $\mathrm{g}$ & $\mathrm{kg}^{-1}$ & , \\
\hline \multicolumn{14}{|c|}{ Latossolo Vermelho-distrófico - Passo Fundo } \\
\hline Floresta & 5,0 & 0,3 & 8,5 & 7,0 & 2,7 & 0,3 & 18,6 & 53,8 & 1 & 10 & 220 & 210 & 560 \\
\hline $\mathrm{PD} T / \mathrm{S}$ & 5,2 & 0,2 & 6,4 & 4,5 & 1,8 & 0,8 & 13,5 & 52,6 & 7 & 10 & 230 & 130 & 630 \\
\hline$P D T / S-E / M$ & 5,2 & 0,3 & 6,6 & 3,4 & 2,1 & 0,7 & 12,8 & 48,4 & 4 & 10 & 230 & 130 & 630 \\
\hline $\mathrm{PC} T / \mathrm{S}$ & 5,3 & 0,2 & 5,2 & 3,7 & 1,5 & 0,6 & 11,0 & 52,7 & 3 & 10 & 230 & 140 & 620 \\
\hline $\mathrm{PC} T / \mathrm{S}-\mathrm{E} / \mathrm{M}$ & 5,6 & 0,2 & 5,3 & 3,4 & 1,9 & 0,6 & 11,1 & 53,2 & 3 & 10 & 230 & 140 & 620 \\
\hline \multicolumn{14}{|c|}{ Latossolo Vermel ho-Amarelo - Santo Antônio de Goiás } \\
\hline Cerrado & 4,8 & 1,2 & 8,8 & 0,5 & 0,2 & 0,1 & 9,6 & 8,1 & 1 & 180 & 130 & 110 & 580 \\
\hline$P D P / A-P / S$ & 5,8 & 0,0 & 3,6 & 1,6 & 0,9 & 0,2 & 6,3 & 43,2 & 9 & 220 & 180 & 100 & 500 \\
\hline $\mathrm{PD} \mathrm{Cr} / \mathrm{A}-\mathrm{Mi} / \mathrm{S}$ & 5,7 & 0,1 & 4,1 & 1,3 & 0,9 & 0,2 & 6,5 & 37,0 & 6 & 210 & 180 & 110 & 500 \\
\hline $\mathrm{PC} P / \mathrm{A}-\mathrm{P} / \mathrm{S}$ & 6,1 & 0,0 & 3,3 & 2,8 & 1,1 & 0,4 & 7,6 & 56,1 & 4 & 200 & 170 & 150 & 480 \\
\hline $\mathrm{PC} \mathrm{Cr} / \mathrm{A}-\mathrm{Mi} / \mathrm{S}$ & 6,1 & 0,0 & 3,1 & 2,5 & 1,2 & 0,4 & 7,2 & 56,9 & 6 & 190 & 170 & 150 & 490 \\
\hline
\end{tabular}

(1) PD: plantio direto; PC: preparo convencional; P: pousio; T: trigo; S: soja; E: ervilhaca; M: milho; A: arroz; Cr: crotalária; Mi: milheto. (2) Determinado na camada de $0-5 \mathrm{~cm}$.

solo foram coletadas nos diferentes sistemas de preparo e rotação de culturas, bem como nas áreas remanescentes de vegetação secundária (floresta e cerrado).

$\mathrm{Na}$ área experimental de Passo Fundo, em cada subparcela de cada bloco, foi feita uma pequena trincheira e, com o uso de uma col her de pedreiro de tamanho médio, fatias de solo no perfil, na profundidade de $0-5 \mathrm{~cm}$, foram transferidas para sacos plásticos. I gual procedimento de col eta foi executado em Santo Antônio de Goiás, onde as amostras foram obtidas em cada sistema de cultivo $(n=3)$ em cada faixa. As amostras de solos foram secas a $40^{\circ} \mathrm{C}$, moídas e peneiradas a $2 \mathrm{~mm}$ para posterior execução das análises. Procedimento similar de col eta foi efetuado nas áreas sob floresta secundária. Entretanto, as trincheiras $(\mathrm{n}=3)$ foram abertas no interior da mata distanciadas em 30 m uma da outra.

As frações leves da matéria orgânica foram obtidas segundo método sugerido por Sohi et al. (2001) . Uma massa de $5 \mathrm{~g}$ de TFSA foi transferida para tubos de centrífuga de $50 \mathrm{~cm}^{3}$, onde foram adicionados $35 \mathrm{~mL}$ de lodeto de Sódio ( $\mathrm{Nal}$ ), a uma densidade de $1,80 \mathrm{~g} \mathrm{~cm}^{-3}$. A pós breve agitação manual, visando permitir que as frações orgânicas não associadas aos componentes minerais do solo atingissem a superfície da suspensão, as amostras foram centrifugadas a 18.000 x g por 15 min para que as partículas minerais do solo permanecessem no fundo do frasco e, por aspiração, coletou-se a fração leve-livre (FLL) presente na superfície da solução de Nal . A FLL, juntamente com soluç̧ão de $\mathrm{Nal}$, foi aspirada para um sistema de filtragem a vácuo (Sistema Asséptico Sterifil, $47 \mathrm{~mm}$ - Millipore) que continha membranas filtrantes lisas constituídas de ésteres inertes de celul ose (Membrana Filtrante MF - Millipore), onde foi separada da sol ução de $\mathrm{Nal}$.

Em seguida, as frações $F L L$ retidas na membrana foram cuidadosamentelavadas com o auxílio de uma pisseta com água destilada, para retirar o excesso de solução de $\mathrm{Nal}$. Ainda com o auxílio de uma pisseta com água destilada, as frações FLL e água destilada foram transferidas para frascos plásticos e congeladas para posterior liofilização.

Após a remoção da FLL, aplicou-se ultrassom (400 $\mathrm{J} \mathrm{mL}^{-1}$ ) por três minutos com intervalos de um segundo na solução de $\mathrm{Nal}$ e solo restante no tubo de centrífuga em banho de gelo, visando minimizar el evação da temperatura na suspensão. Após o tratamento com ultrassom, as amostras foram novamente centrifugadas a $18.000 \times \mathrm{g}$ por $15 \mathrm{~min}$, obtendo-se assim a fraçãoleveintra-agregado (FLI).

Os diferentes grupos funcionais das frações leveslivres $(F L L)$ e intra-agregado ( $F L I)$ foram identificados pela técnica de espectroscopia de infravermel ho com transformada deFourier (IV-TF). Os espectros foram obtidos em triplicata eanalisados conforme freqüência e intensidade das bandas de absorção correspondentes às vibrações de 
estiramento (v) ou deformação angular $(\delta)$, descritas por Colthup et al. (1964), Baes \& Bloom (1989), Silverstein (1994), Stevenson (1994) e Canellas (1999). As medidas foram efetuadas utilizando uma massa de $1 \mathrm{mg}$ de cada fração obtida, misturadas a pastilhas de $100 \mathrm{mg}$ de $\mathrm{KBr}$ sólido. Os espectros foram obtidos na faixa do espectro correspondente a $600 \mathrm{~cm}^{-1}$ a $4.000 \mathrm{~cm}^{-1}$.

Antes da leitura dos espectros da matéria orgânica, foram obtidos espectros do $\mathrm{KBr}$ para identificar possíveis alargamentos na região de $3.400 \mathrm{~cm}^{-1}$, decorrentes da contaminação com água. A caracterização das frações leves por infravermel ho foi realizada por meio da análise das principais bandas de absorção encontradas.

Com base nos espectros de infravermelho adquiridos, foram determinados índices que possibilitaram a identificação de alterações na matéria orgânica das distintas frações leves, decorrentes do processo de humificação e da influência dos sistemas de manejo. A relação entre a absor bância da banda de absorção em $2.929 \mathrm{~cm}^{-1} \mathrm{e}$ em $1.050 \mathrm{~cm}^{-1}$ foi chamada, nestetrabalho, deíndice de hidrofobicidade $(\mathrm{IH})$, uma vez que expressa a relação entre grupos apolares $\left(\mathrm{CH}_{3}\right)$ e polares (-OH, C-O). J á a relação entre 1.660 e $2.929 \mathrm{~cm}^{-1}$, denominada índice de condensação (IC), expressa a proporção de grupos conjugados e alifáticos. Os grupamentos $-\mathrm{CH}_{3}$ em cadeias alquílicas são caracteristicamente hidrofóbicos e recal citrantes. Assim, quantomaior olH de uma molécula orgânica, maior deverá ser sua resistência à degradação microbiana. O índice de condensação (IC) foi utilizado para estabelecer uma análise indireta do grau de condensação/humificação da molécula.

\section{RESULTADOS E DISCUSSÃO}

Os espectros de infravermel ho das frações (levelivre e intra-agregado) apresentaram feição semelhante, o que se deve à influência dominante de resíduos vegetais sobre estas frações (Skjemstad et al., 1986; Golchin et al., 1994a). Contudo, os espectros IV da FLI apresentaram maior sobreposição debandas deabsorção, além de diferenças acentuadas entre os distintos sistemas de preparo do solo.

$\mathrm{Na}$ região espectral de 3.700 a $3.000 \mathrm{~cm}^{-1}$, correspondente às vibrações de estiramento (v) dos grupamentos $-\mathrm{OH}$ e $-\mathrm{NH}$, os espectros da fração leve-livre apresentaram bandas de absorção mais nítidas, permitindo a visualização de funções nitrogenadas. Nos espectros de FLL de amostras de Passo F undo, a banda de absorção O-H $\left(3.320 \mathrm{~cm}^{-1}\right)$ é forte e alargada, conseqüência da vibração em vários níveis energéticos de grupamentos -OH em pontes de hidrogênio inter e intramoleculares (Figura 1). Nesta região espectral, os espectros da fração leve intra-agregado em Latossolo de Passo
Fundo sob floresta e PD foram muito semelhantes aos obtidos na fração leve-livre (Figura 2). Entretanto, na FLI de sol o sob PC, observou-se uma única banda de absorção al argada e sobreposta, que está deslocada para um nível superior de energia $\left(3.463 \mathrm{~cm}^{-1}\right)$. Tal característica indica uma configuração mais rígida das ligações $\mathrm{O}-\mathrm{H}$ e N-H, pela formação mais intensa de pontes de hidrogênio inter eintramoleculares, o que possivel mentese deve à maior complexidade estrutural da FLI neste sistema de preparo.

A FLL de Latossolo de Santo Antônio de Goiás apresentou espectro com bandas de absorção N-H (3.620 e $\left.3.526 \mathrm{~cm}^{-1}\right)$ e O-H $\left(3.446 \mathrm{~cm}^{-1}\right)$ estreitas e com maior energia de vibração, características de ligações livres (Figura 1). Os espectros de FLI de sol o sob cerrado e PD, todavia, apresentaram bandas de absorção menos evidentes (Figura 2). Semel hanteao observado em Passo F undo, as FLI desol o sob aração e gradagem também sofreram alterações na configuração estrutural de -OH.

Os grupamentos alifáticos $-\mathrm{CH}_{3}\left(2.929 \mathrm{~cm}^{-1}\right)$ e- $\mathrm{CH}_{2}$ $\left(2.854 \mathrm{~cm}^{-1}\right)$ apresentaram variações pronunciadas de acordo com o tipo de preparo do solo. As bandas de absorção correspondentes às frações leves-livres e intra-agregado de sol o sob vegetação natural ePD mostraram-se mais definidas, quando comparadas às amostras de PC em ambas as áreas estudadas.

Todas as frações leves apresentaram banda de absorção $C=C$ de aromáticos $\left(1.489 \mathrm{~cm}^{-1}\right)$ pouco pronunciada, característica inerente aos compartimentos lábeis da matéria orgânica. A banda de absorção em $1.647 \mathrm{~cm}^{-1}$, que pode ser atribuída à presença de duplas ligações conjugadas, é mais acentuada na fração leve intra-agregado (Figura 2). O aumento de duplas ligações conjugadas indica maior condensação das moléculas, caracterizando um material mais humificado (Stevenson, 1994).

A região de absorção deestiramentos $\mathrm{C}$-O deéster e O-H alcoólico (1.300-1.000 $\left.\mathrm{cm}^{-1}\right)$, geralmente atribuída à presença de polissacarídeos na MOS, seria a mais peculiar para um composto orgânico, podendo ser utilizada para comparações no processo de humificação da MOS. A FLI apresentou bandas de absorção C-O de menor intensidade do quea FLL em ambas as áreas e em todos os tratamentos estudados. As bandas de absorção de pol issacarídeos nas frações leves intra-agregado em preparo convencional apresentaram menor intensidade em relação às bandas de absorção observadas nas FLI dos solos sob vegetação natural e plantio direto, revel ando maior al teração destas frações, com perda de polissacarídeos, que pode ser atribuída a um maior grau de humificação.

A região espectral de 900 a $650 \mathrm{~cm}^{-1}$ pode expressar o grau de substituição de átomos nas ligações de grupamentos aromáticos (Nakanishi, 1962). Tomando as frações leves de solos sob vegetação natural como referência, as diferenças 


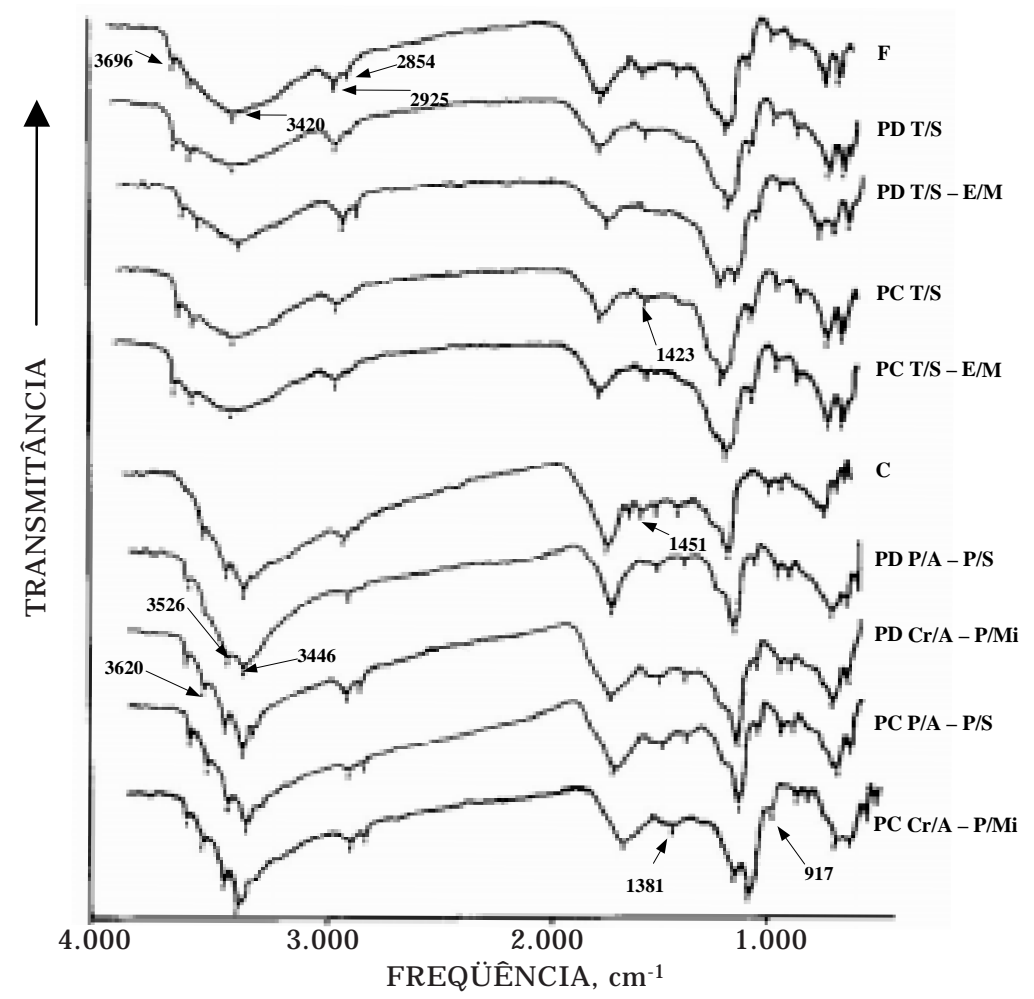

Figura 1. E spectros de infravermelho da fração leve-livre. F: Latossolo sob floresta em Passo F undo; PD: plantio direto; T: trigo, S: soja; E: ervilhaca; M: milho; PC: preparo convencional; C: Latossolo sob Cerrado em Sto. Anto de Goiás; P: pousio; A: arroz; Cr: crotalária; Mi: mi lheto.

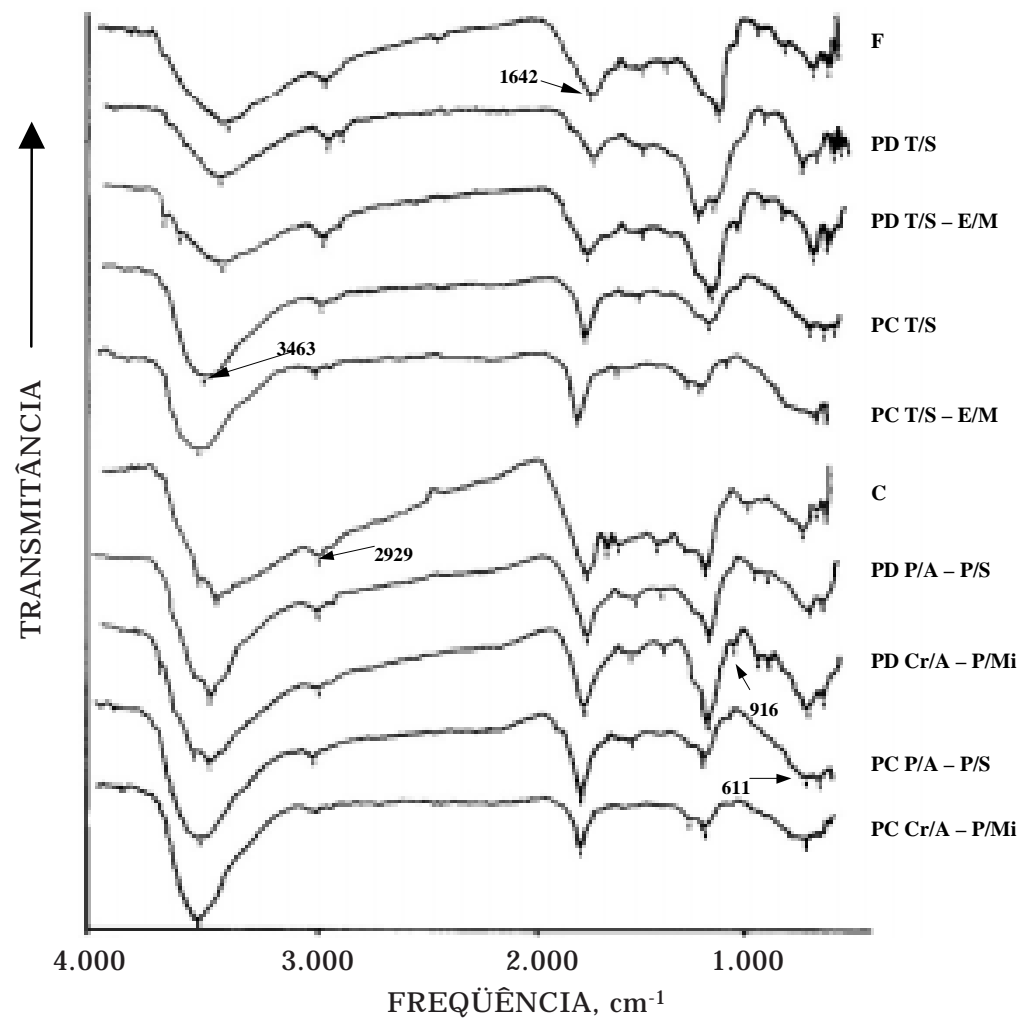

Figura 2. Espectros de infravermelho da fração leve intra-agregado. $F$ : Latossolo sob floresta em Passo Fundo; PD: plantio direto; T: trigo, S: soja; E: ervilhaca; M: milho; PC: preparo convencional; C: Latossolo sob Cerrado em Sto. Anto de Goiás; P: pousio; A: arroz; Cr: crotalária; Mi: milheto. 
encontradas nesta região mostram a influência do tipo de preparo do sol o sobre a matéria orgânica. Os espectros IV da fração leve-livre, nos solos de Passo Fundo e Santo Antônio de Goiás, apresentaram feição semel hante para todos os tratamentos avaliados (Figura 1). Os grupamentos aromáticos da FLI em solos sob PC, em ambas as áreas estudadas, apresentaram mai or grau de substituição do que o observado para as mesmas frações em sol o sob vegetação natural e PD (Figura 2), indicando maior transformação desta fração em sol os sob preparo convencional.

Os índices de hidrofobi ci dade e condensação para as frações leves-livres e intra-agregado dos sol os de Passo F undo estão apresentados no quadro 1. Não foram observadas diferenças significativas entre os distintos sistemas de manejo, entretanto a FLI apresentou valores de IH e IC significativamente superiores aos observados para a FLL. Os índices encontrados para as frações leves dos sol os de Santo Antônio de Goiás seguiram o mesmo padrão observado em Passo Fundo (Quadro 2). Não foram observadas diferenças significativas entre os distintos tratamentos, mas os índices das frações leves intra-agregado superaram aquel es das frações leves-livres.

A análise dos índices de hidrofobicidade e condensação das frações leves revela que a FLI apresenta-semais humificada quea FLL, por mostrar maior proporção de grupamentos recalcitrantes e maior condensação. O aumento da proporção de grupamentos $-\mathrm{CH}_{3}$ na FLI constitui o resultado da utilização de carboi dratos facilmente decomponíveis pelos microrganismos e a preservação seletiva do carbono mais recalcitrante, durante o processo de decomposição da matéria orgânica e de agregação do solo. (Ladd et al., 1993; Golchin et al., 1994a; Golchin et al., 1994b; Zech et al., 1997).

A análise espectroscópica de infravermelho possibilitou a identificação de grandes diferenças estruturais entre as frações leves-livres e intraagregado. A FLI apresentou espectros com bandas de absorção menos intensas e mais sobrepostas do que a FLL, em virtude da maior alteração em sua estrutura, em decorrência do processo dehumificação.

Quadro 2. Índices de hidrofobicidade (IH) e de condensação (IC) para as frações leves-livres(FLL) e intra-agregado (FLI) na camada de 0-5 cm de solo em Passo Fundo ${ }^{(1)}$

\begin{tabular}{|c|c|c|c|c|}
\hline \multirow[b]{2}{*}{ Tratamento ${ }^{(2)}$} & \multicolumn{2}{|c|}{ IH } & \multicolumn{2}{|c|}{ IC } \\
\hline & F L L & F LI & F L L & F LI \\
\hline Mata & $0,47(0,06)$ & $0,63(0,09)$ & $1,13(0,02)$ & $1,16(0,03)$ \\
\hline $\mathrm{PD} T / \mathrm{S}$ & $0,38(0,09)$ & $0,54(0,05)$ & $1,10(0,02)$ & $1,17(0,10)$ \\
\hline PD T/S-E/M & $0,32(0,02)$ & $0,49(0,16)$ & $1,07(0,09)$ & $1,15(0,08)$ \\
\hline $\mathrm{PC} T / \mathrm{S}$ & $0,38(0,04)$ & $0,63(0,23)$ & $1,09(0,03)$ & $1,18(0,15)$ \\
\hline $\mathrm{PC} \mathrm{T/S-E/M}$ & $0,40(0,10)$ & $0,67(0,22)$ & $1,07(0,03)$ & $1,18(0,28)$ \\
\hline Médias(3) & $0,39(0,08)$ & $0,59(0,16)$ & $1,10(0,04)$ & $1,17(0,13)$ \\
\hline
\end{tabular}

(1) Valores entre parênteses referem-se ao erro-padrão da média. ${ }^{(2)}$ PD: plantio direto; PC: plantio convencional; T: trigo; S: soja; E: ervilhaca; $M$ : milho. ${ }^{(3)}$ Médias diferem estatisticamente pelo teste t de Student, $p<0,05$.

Quadro 3. Índices de hidrofobicidade (IH) e de condensação (IC) para as frações leves (FLL e FLI) na camada de 0-5 cm de solo em Santo Antônio de Goiás ${ }^{(1)}$

\begin{tabular}{|c|c|c|c|c|}
\hline \multirow{2}{*}{ Tratamento(2) } & \multicolumn{2}{|c|}{ IH } & \multicolumn{2}{|c|}{ IC } \\
\hline & F LL & F LI & F L L & F LI \\
\hline Mata & $0,70(0,10)$ & $0,88(0,01)$ & $1,13(0,01)$ & $1,28(0,01)$ \\
\hline $\mathrm{PD} T / \mathrm{S}$ & $0,61(0,09)$ & $0,84(0,07)$ & $1,11(0,07)$ & $1,54(0,34)$ \\
\hline PD T/S-E/M & $0,56(0,16)$ & $0,79(0,05)$ & $1,05(0,05)$ & $1,17(0,11)$ \\
\hline $\mathrm{PCT} / \mathrm{S}$ & $0,52(0,09)$ & $0,93(0,02)$ & $1,03(0,02)$ & $1,61(0,57)$ \\
\hline $\mathrm{PC}$ T/S-E/M & $0,47(0,04)$ & $0,72(0,03)$ & $1,01(0,03)$ & $1,15(0,07)$ \\
\hline Médias(3) & $0,57(0,12)$ & $0,83(0,15)$ & $1,07(0,06)$ & $1,35(0,32)$ \\
\hline
\end{tabular}

(1) Valores entre parênteses referem-se ao erro-padrão da média. (2) PD: plantio direto; PC: plantio convencional; T: trigo; S: soja; E: ervilhaca; M: milho. (3) Médias diferem estatisticamente pelo teste te Student, $p<0,05$. 
O aumento da condensação e a redução de polissacarídeos seriam conseqüências deste processo. As diferenças na composição el ementar destas distintas frações (relação C/N), observadas por Freixo (2000), confirmam os resultados obtidos por infravermel ho.

\section{CONCLUSÕES}

1. As análises espectroscópicas por infravermelho indicam queas frações leves-livres e intra-agregado constituem dois compartimentos distintos da matéria orgânica do solo.

2. A fração leve intra-agregado apresentou espectros com bandas de absorção mais sobrepostas doquea fraçãolevelivre, indicandomaior transformação de sua estrutura em decorrência do processo de humificação.

3. O tipo de preparo do sol oe a rotação de culturas não promoveram al terações na natureza química da fraçãoleve-livreem ambos os Latossol os dePasso Fundo e de Santo Antônio de Goiás.

4. A fração leve intra-agregado sofreu maior al teração estrutural em sol os sob preparo convencional.

5. Osíndices dehidrofobi cidade (IH) econdensação (IC) foram superiores na fraçãoleve intra-agregado, caracterizando sua maior recalcitrância e condensação.

\section{AGRADECIMENTOS}

À F undação Coordenação deA perfei çoamento de Pessoal de Nível Superior (CAPES) e à Fundação Carlos Chagas Filho deAmparoà Pesquisa do Estado do Rio de J aneiro (FAPERJ ), pelo auxílio financeiro à pesquisa; ao Dr. Cleber M. Guimarães (Embrapa Arroz e Feijão) e Dr. Henrique P. dos Santos (Embrapa Trigo), por disponibilizarem os experimentos de campo para a coleta de amostras de solos; ao Sr. Eli, técnico do I nstituto de Química da UFRRJ, pela confecção dos espectros de infravermel ho, eao professor Fernando Guridi, pelas sugestões na redação deste trabal ho.

\section{LITE RATURA CITADA}

BAES, A.U. \& BLOOM, P.R. Diffuse reflectance and transmission Fourier transform infrared (DRIFT) spectroscopy of humic and fulvic acids. Soil Sci. Soc. Am. J ., 53:695-700, 1989.

CABANISS, S.E. Carboxilic acid content of a fulvic acid determined by potentiometric and aqueous Fouriertransform infrared spectrometry. Anal. Chim. Acta, 255:2330. 1991.
CANELLAS, L.P. Avaliação de características fisico-químicas de ácidos húmicos. Seropédica, Universidade Federal Rural do Rio de J aneiro, 1999. 164p. (Tese de Doutorado)

CELI, L.; SCHNITZER, M.\& NÈGRE, M. Analysis of carboxyl groups in soil humic acids by a wet chemical method, Fourier-transform infrared spectrophotometry, and solution-state carbon-13 nuclear magnetic ressonance: a comparative study. Soil Sci. 162:189-197.

CHRISTENSEN, B.T. Physical fractionation of soil and organic matter in primary particle size and density separates. Adv. Soil Sci., 20:1-90, 1992.

CHRISTENSEN, B.T. Matching measurable soil organic matter fractions with conceptual pools in simulation models of carbon turnover: revision of model structure. In: POWLSON, D.S.; SMITH, P. \& SMITH, J.V., eds. Evaluation of soil organic matter models. NATO ASI Series, VolumeI, 38, Berlin, Springer-Verlag. p.143-159. 1996.

COLTHUP, N.B.; DALY, L.H. \& WIBERLEY, S.E. Introduction to infrared and raman spectroscopy. New York, Academic Press, 1964. 511p.

DAVIS, W.M.; ERICSON, C.L.; J OHNSTON, C.T.; DELFINO, J J . $\&$ PORTER, J.E. Quantitative Fourier-transform infrared spectroscopic investigation of humic substance functional group composition. Chemosphere, 38:2913-2928. 1999.

FELLER, C. Organo-mineral interactions in tropical soils - In search of "functional" organic matter pools: the particlesize fractionation approach. In: ENCONTRO BRASI LEIRO DE SUBSTÂNCIAS HÚMICAS, 3., Santa Maria, 1999. Anais. Santa Maria, Universidade Federal deSanta Maria, 1999. p.104-124.

FELLER, C. \& BEARE, M.H. Physical control of soil organic matter dynamics in the tropics. Geoderma, 79:69-116, 1997.

FREITAS, P.L.; BLANCANEAUX, P.; GAVINELLI, E.; LARRÉLARROUY, M.C.\& FELLER, C. Nível enatureza do estoque orgânico de Latossolos sob diferentes sistemas de uso e manejo. Pesq. Agropec. Bras., 35:157-170, 2000.

FREIXO, A.A. Caracterização da matéria orgânica de Latossolos sob diferentes sistemas de cultivo através de fracionamento físico e espectroscopia de infravermelho. Seropédica, Universidade Federal Rural do Rio de J aneiro, 2000. 86p. (Tese de Mestrado)

GOLCHIN, A.; OADES, J.M.; SKJ EMSTAD, J .O. \& CLARKE, P. Study of free and occluded particulate organic matter in soils by solid state ${ }^{13} \mathrm{C}$ CP/MAS NMR spectroscopy and scanning el ectron microscopy. Aust. J . Soil Res., 32:285-309, 1994a.

GOLCHIN, A.; OADES, J .M.; SKJ EMSTAD, J .O. \& CLARKE, P. Soil structure and carbon cycling. Soil Biol. Biochem., 32:1043-1068, 1994b.

INBAR, Y.; CHEN, Y. \& HADAR, Y. Solid-state carbon-13 nuclear magnetic resonance and infrared spectroscopy of composted organic matter. Soil Sci. Soc. Am. J ., 53:1695-1701. 1989.

J OHNSTON, C.T.; DAVIS, W.M.; ERICKSON, C. \& DELFINO, J.J. Characterization of humic substances using Fourier transform infrared spectroscopy. I n: SENESI, N. \& MIANO, T.M., eds., Humic substances in the gl obal environment and implications on human health. New York: Elsevier Scientific Publ. Co., 1994. p.145-152. 
J OHNSTON, C.T. \& AOCHI, Y.O. Fourier transform infrared and raman spectroscopy. In: SPARKS, D.L., ed. Methods of soil analysis. Chemical methods. 2.ed. Madison, American Society of Agronomy. 1996. p. 269-321. (SSSA Book Series, 5)

LADD, J .N.; FOSTER, R.C. \& SKJ EMSTAD, J .O. Soil structure: carbon and nitrogen metabolism. Geoderma, 56:401-434, 1993.

MARTIN-NETO, L.; CRUVINEL, P.E.; MATTOSO, L.H.C. \& COLNAGO, L.A. Espectroscopias de infravermelho, ultravioleta-visível e pixe: alguns resultados disponíveis. In: CRESTANA, S.; CRUVINEL, P.E.; MASCARENHAS, S. \& BISCEGLI, C.I., eds. Instrumentação agropecuária: contribuições nolimiar do novo século. São Carlos, Empresa Brasileira de Pesquisa Agropecuária, 1996. p.51-90.

NAKANISHI, K. Infrared absorption spectroscopy. Tokyo, Nankondo Company, 1962. 233p.

PARTON, W.J .; SCHIMEL, D.S. \& COLE, C.V. Analysis of factors controlling soil organic matter levels in great plains grasslands. Soil Sci. Soc. Am. J., 51:1173-1179, 1987.

SCHNITZER, M. Characterization of humic constituents by spectroscopy. In: MCLARE N, A.D. \& SKUJ INS, J ., eds. Soil Biochemistry, V.2, New York: Marcel-Decker. p. 60-95. 1971.

SILVA, C.A.; ANDERSON, S.J . \& VALE, F.R. Carbono, nitrogênio e enxofre em frações granulométricas de dois Latossolos submetidos à calagem e adubação fosfatada. R. Bras. Ci. Solo, 23:593-602, 1999.
SILVERSTEIN, R.M.; BASSLER, G. \& MORRILL,T.C. I dentificação espectrométrica de compostos orgânicos. Rio de J aneiro, Guanabara-Koogan, 1994. 387p.

SIX, J., ELLIOTT, E.T. \& PAUSTIAN, K. Soil macroaggregate turnover and microaggregate formation: a mechanism for C sequestration under no-tillage agriculture. Soil Biol. Biochem., 32:2099-2103, 2000

SKJ EMSTAD, J .O.; DALAL, R.C. \& BARRON, P.F. Spectroscopic investigations of cultivation effects on organic matter of vertisols. Soil Sci. Soc. Am. J ., 50:354-359, 1986.

SOHI, S.; MAHIEU, N.; ARAH, J.R.M.; POWLSON, D.S.; MADARI, B. \& GAUNT, J.L. A procedure for isolating soil organic matter fractions suitable for modelling. Soil Sci. Soc. Am. J ., 65:1121-1128, 2001.

STEVENSON, F.J . Humus chemistry: genesis, composition, reactions. 2.ed. New York, J ohn Wiley \& Sons, 1994. 496p.

THENG, B.K.G.; TATE, KR. \& SOLLINS, P. Constituents of organic matter in temperate and tropical soils. In: COLEMAN, D.C.; OADES, J.M. \& UEHARA, G., eds. Dynamics of soil organic matter in tropical ecossystems. Honolulu, University of Hawaii Press, 1989. p.5-32.

WANDER, M.M. \& TRAINA, S.J . Organic matter fractions from organically and conventionally managed soils: II. Characterization of composition. Soil Sci. Soc. Am. J., 60:1087-1094, 1996.

ZECH, W.; SENESI, N.; GUGGENBERGER, G.; KAISER, K.; LEHMANN, J .; MIANO, T.M.; MILTNER, A. \& SCHROTH, G. Factors controlling humification and mineralization of soil organic matter in the tropics. Geoderma, 79:117-161, 1997. 
A.A. FREIXO et al.

R. Bras. Ci. Solo, 26:445-453, 2002 